\section{Left lower limb deep venous thrombosis, May-Thurner syndrome and endovascular management}

\author{
Khawaja B. Wabeed, MBBS, FCPS, \\ Hassan R. Mohammed, MBBS, MRCS, KhaledS. Salem, MBBS, FRCR, \\ Mohamed A. Shaltout, MD, FRCS, Ali S. Alshehri, MBBS, SB-RAD, \\ EmadF. Said, MBBS, FEBR,AbdulhadiS. Almubarak, GSCE, MBBS, \\ Zechariah J. Arulanantham, $M S c, P h D$.
}

\section{ABSTRACT}

Objectives: To determine the prevalence of May-Thurner syndrome (MTS) in left lower limb deep venous thrombosis (DVT) cases and to analyze the outcome of endovascular intervention in these patients.

Methods: A record-based descriptive study was carried out in Radiology Department, King Fahad Military Medical Complex, Dhahran, Saudi Arabia, including patients who underwent lower limb duplex ultrasounds between January 2015-2021. Patients with bilateral DVTs, known pelvic masses, and pelvic surgeries were excluded. All patients positive for DVTs were identified and further imaging was reviewed. Left common iliac vein compression of $50 \%$ or more on computed tomography (CT) was considered positive for MTS. Endovascular interventions (venoplasty alone or with stenting) were evaluated and success recorded by observing patency of vein on follow-up imaging or improvement of symptoms on follow-up visits.

Results: Of 182 patients with left lower limb duplex studies, 51 patients were positive for DVTs. A total of 37 patients had CTs and 21 patients had MTS (17 females, 3 males). A total of 15 patients underwent endovascular interventions, 2 patients had venoplasties alone (one successful) and 13 patients had venoplasties with stenting (10 successful).

Conclusion: Patients with MTS as cause of DVT may benefit from early endovascular intervention.

Keywords: May-Thurner syndrome, iliac vein compression syndrome, venoplasty, endovascular

Saudi Med J 2022; Vol. 43 (1): 108-112 doi: 10.15537/smj.2022.43.1.20210473

$\mathrm{D}$ eep venous thrombosis (DVT) is a common clinical condition with both morbidity and mortality due to its complications related to pulmonary embolism. ${ }^{1}$ Timely diagnosis and early management are needed to avoid complications. Anticoagulation is a mainstay of treatment. ${ }^{2}$ However, when DVT is caused by an anatomic or structural abnormality, medical treatment may not be sufficient. May-Thurner syndrome (MTS), also called Cockett syndrome or iliac vein compression syndrome, is characterized by the compression of left common iliac vein by the right common iliac artery over the fourth lumbar vertebra. ${ }^{1,2}$ Rudolph Virchow in 1851 reported increased incidence of left iliac vein thrombosis in cadavers having positive left iliofemoral thrombosis. ${ }^{3}$ Later on, May and Thurner reported intraluminal fibrous bands secondary to vein compression by the artery in nearly one-fourth (22\%) of 430 cadaver cases in $1957 .{ }^{4}$ Endovascular management was considered a preferrable approach to dissolve thrombus and also to treat underlying compression by stent replacement. ${ }^{5}$

The true prevalence of MTS is underestimated. ${ }^{6}$ More recently, because of the availability of modern imaging techniques and improved visualization of the iliac veins, MTS is now more frequently diagnosed.? We did not find any study in literature describing the prevalence of MTS in patients with lower limb DVTs in Saudi Arabia. Therefore, this study aims to estimate the prevalence of MTS in patients with left lower limb DVTs, its diagnostic imaging criteria on computed tomography (CT), and the role of endovascular management.

Methods. A record-based descriptive study was carried out in the Radiology Department, King Fahad Military Medical Complex, Dhahran, Saudi Arabia, including all Saudi patients who underwent left lower limb duplex ultrasound studies for DVTs between January 2015-2021. Patients with bilateral DVTs, known pelvic masses, pelvic surgeries, and limited studies were excluded. The study was approved by the Hospital Research and Ethics Committee and was conducted in accordance with the Helsinki Declaration.

Clinical information was obtained from patients' medical records via Hospital Information System (HIS), while CTs and intervention related findings were reviewed through Radiology Information System/ Picture Archiving and Communication System. All clinical and radiological data were kept strictly confidential.

Duplex ultrasounds of lower limbs for DVTs were carried out by experienced technicians under the supervision of radiologists on a properly maintained and calibrated Toshiba machine (Aplio MX 2010, Japan) using a $7.5 \mathrm{MHz}$ linear probe. Computed tomography scans were carried out on a 128-slice CT machine (SOMATOM Definition Flash, Siemens Healthcare, Germany), and CT studies were obtained in 
either portovenous or delayed (venogram) phases after intravenous administration of water-soluble contrast (Iomeron ${ }^{\circledR} 350$, GE Healthcare, Cork, Ireland).

A first or single episode of DVT was considered as acute DVT while recurrent (repeated episodes) or long standing (more than 6 months) history of non-resolved DVT was taken as chronic DVT. All patients positive for (acute or chronic) DVTs were identified, and further CT imaging was reviewed. Imaging criterion of left common iliac vein compression by $50 \%$ or more on CT was considered positive for MTS. Two experienced radiologists (with more than 10 years of general or body imaging) reviewed the CT scans and were kept blinded of clinical information. Good inter-observer agreement was noted (with Cohen's kappa value of 0.79). Mutual consensus reading was carried out for minor discrepancies. Endovascular interventions (venoplasties alone or with stenting) were carried out by experienced vascular surgeons under fluoroscopy and results evaluated with success were documented by observing patency of the vein on follow-up imaging or improvement of symptoms on follow-up visits.

Statistical analysis. Age and gender of all patients were recorded. Risk factors, clinical presentations, intervention procedures and outcomes were evaluated and compared. Data was collected and analyzed using Statistical Package for the Social Sciences for Windows, version 22 (IBM Corp., Armonk, NY, USA).

Results. Of 182 patients who underwent left lower limb Doppler studies, 51 (28\%) patients were positive for left sided DVTs. A total of 37 (72\%) patients had CT imaging available and 21 patients had MTS (17 females and 4 males). Majority of the patients $(n=21)$ had portovenous phase CT imaging only, while the remaining had delayed available venograms (Figure 1).

A total of 15 patients underwent endovascular interventions (Table 1). Two patients had venoplasties alone (one successful, success $=50 \%$ ) and 13 patients had venoplasties with stenting (10 successful, success $=77 \%$ ) (Figure 2). Three patients were having other risk factors namely, factor V Leiden, protein S deficiency, and antiphospholipid syndrome. One patient of failed stenting had in-stent restenosis or stent thrombosis.

Discussion. May-Thurner syndrome is a cause of DVT and the symptoms depend on the degree of vein

Disclosure. Authors have no conflict of interests, and the work was not supported or funded by any drug company.

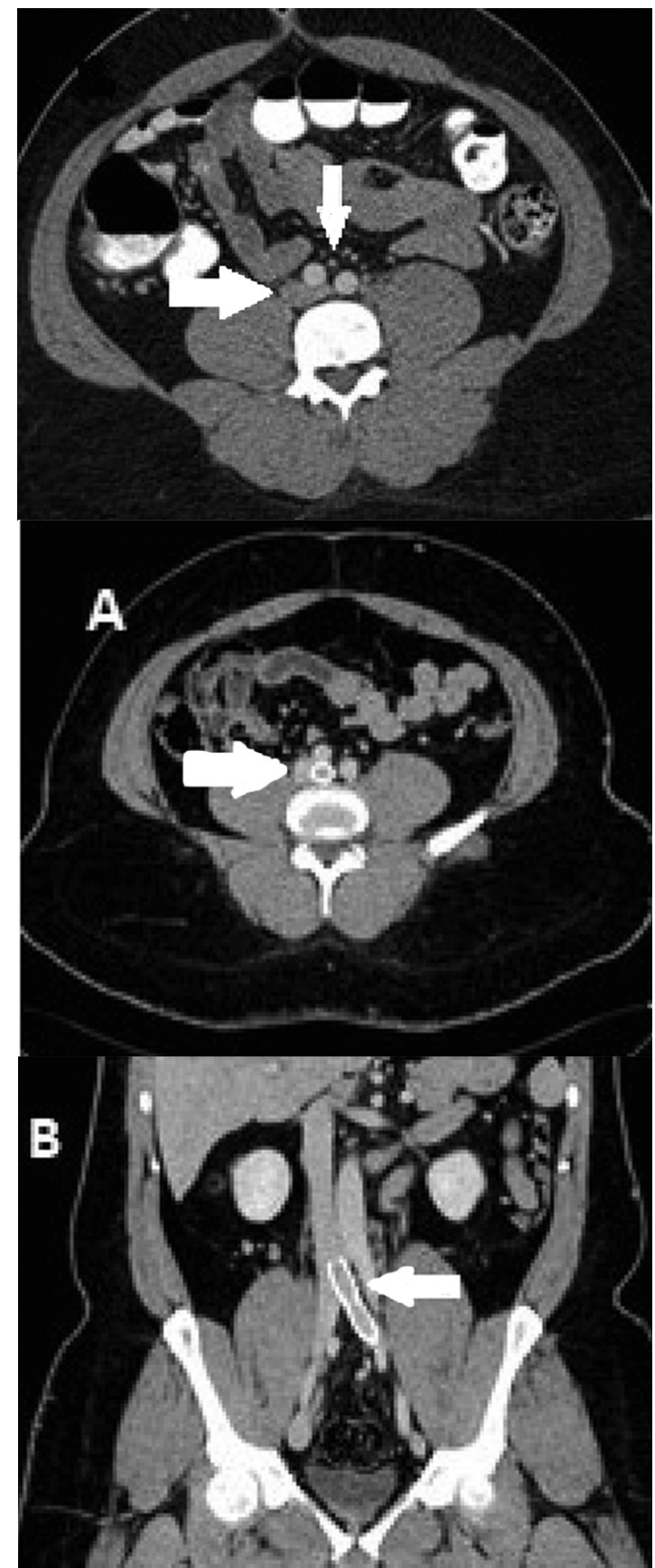

Figure 1 - Selected axial portovenous phase computed temography (CT) image showing left common iliac vein (horizontal arrow) compressed by the right common iliac artery (vertical arrow). Selected A) axial and B) coronal portovenous phase CT images showing left common iliac vein stent in-position.

compression. It is characterized by varying degrees of venous hypertension (pain, swelling, and claudication) and can combine with acute DVT, recurrent DVT or post thrombotic syndrome (signs and symptoms of 
Table 1 - Endovascular management and its outcome in May-Thurner syndrome positive and intervened patients.

\begin{tabular}{|c|c|c|c|c|c|c|}
\hline Patient no. & Age (years) & Gender & Presentation & Risk factor (hematological) & Intervention (endovascular) & Outcome \\
\hline 1 & 34 & Female & Chronic & Positive & Angioplasty alone & Failed \\
\hline 2 & 25 & Female & Chronic & Negative & Angioplasty + stent & Success \\
\hline 3 & 27 & Female & Acute & Negative & Angioplasty + stent & Success \\
\hline 4 & 22 & Female & Acute & Negative & Angioplasty + stent & Success \\
\hline 5 & 48 & Male & Chronic & Negative & Angioplasty alone & Success \\
\hline 6 & 36 & Female & Acute & Negative & Angioplasty + stent & Failed \\
\hline 7 & 48 & Male & Chronic & Negative & Angioplasty + stent & Success \\
\hline 8 & 41 & Female & Chronic & Negative & Angioplasty + stent & Success \\
\hline 9 & 21 & Female & Chronic & Positive & Angioplasty + stent & Failed \\
\hline 10 & 29 & Male & Acute & Negative & Angioplasty + stent & Success \\
\hline 11 & 69 & Female & Chronic & Negative & Angioplasty + stent & Success \\
\hline 12 & 22 & Female & Chronic & Negative & Angioplasty + stent & Success \\
\hline 13 & 39 & Female & Chronic & Negative & Angioplasty + stent & Success \\
\hline 14 & 22 & Female & Chronic & Positive & Angioplasty + stent & Failed \\
\hline 15 & 48 & Male & Chronic & Negative & Angioplasty + stent & Success \\
\hline
\end{tabular}
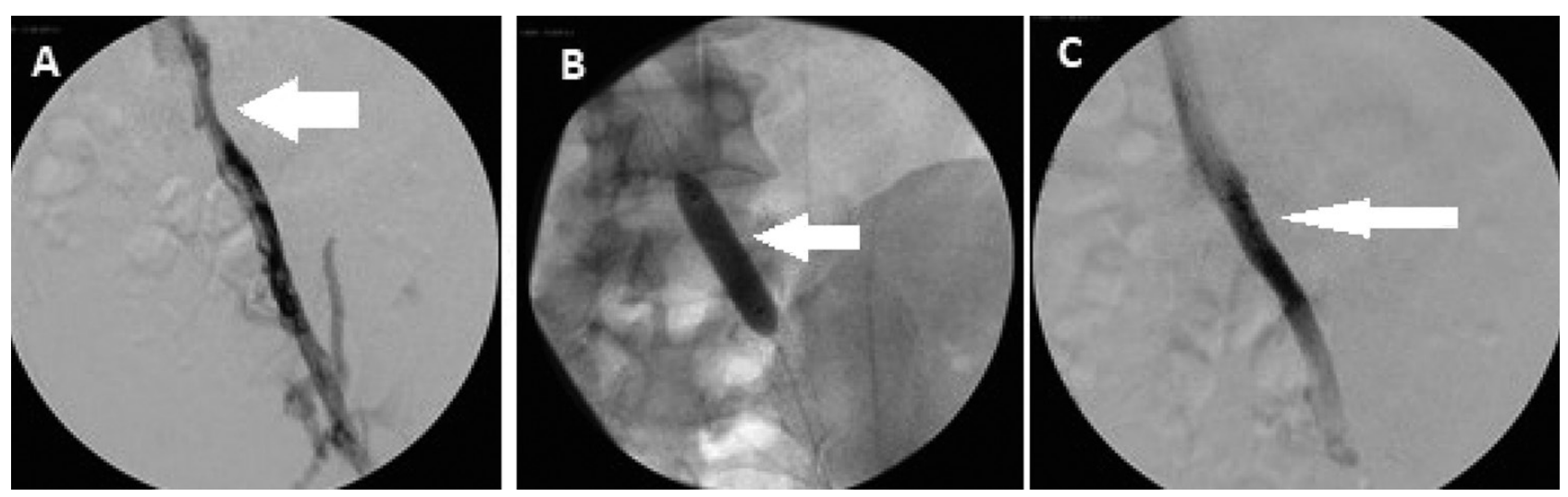

Figure 2 - Selected spot digital subtraction images showing: focal narrowing along the proximal left common A) iliac vein, B) balloon inflation, and C) subsequent stent placement.

chronic venous insufficiency). ${ }^{8}$ This study depicted important clinical implications towards the diagnostic and management approach of MTS. Firstly, it was found that most of positive MTS cases were females $(\mathrm{n}=17 ; 81 \%)$ and less than a third $(\mathrm{n}=5)$ of patients had pulmonary embolism event during the course of DVT and related admissions in a period of 2-5 years of initial diagnoses. More than half $(15 / 21)$ of the patients opted for the endovascular interventions, one-third improved by medical management while the rest of the cases refused interventions. Secondly, the majority (more than two-thirds) of the endovascular interventions were found to be successful when followed until a period of 2 years after procedures. Those who had successful outcomes, the mean duration of intervention from first episode of DVT was 3 years, and those who had failures was 8 years. This strongly depicts that the earlier the intervention was carried out, the better the results would be. This study results tally to internationally published reports like that of Kaltenmeier et $\mathrm{al},{ }^{9}$ who presented a systematic review and included 1569 patients from the selected articles. They found MTS more in females and in younger age group presenting with leg swelling and leg pain. Also, they found higher incidence of pulmonary embolism at presentations in such MTS positive patients. Most of patients (approximately 85\%) were treated with endovascular intervention (without or with thrombolysis), while remaining ones were treated with either medical or surgical approach. ${ }^{?}$

Although we did not include younger patients (less than 18 years) in this study. However, reports are available regarding results of endovascular management in such age groups. Hansrani et al, ${ }^{2}$ studied MTS and its endovascular management while reviewing published 
reports of 22 patients under the ages of 18 years, with nearly one-third (35\%) benefitting from thrombolysis (pharmaco-mechanical and catheter induced) and stenting. ${ }^{2}$ They also found more than a third of patients with additional risk factors precipitating thrombosis. Sedhai et $a l,{ }^{10}$ presented a single center review of 6 MTS patients with endovascular therapy and treated those patients successfully with catheter directed thrombolysis and venous stenting. However, they observed stent thrombosis after a 2-year follow-up in 2 of the patients, one having hypercoagulable (antiphospholipid antibody) syndrome and the other with thrombophilia. They stressed upon further investigation in those groups. Atypical presentation of MTS was also known and may include sciatic neuralgia or pelvic congestion and may occur in patients with pelvic kidney, voluminous uterine fibroid, pregnancy, and in post-partum women. ${ }^{11-13}$ Also, right sided MTS may occur, if inferior vena cava is on the left side or if right sided common iliac vein is compressed by branch of right common iliac artery. Limited studies were available to document the incidence of in-stent restenosis and found a disease recurrence in more than a third (30-40\%) of patients and advocated a preprocedural poor inflow and outflow to be associated with recurrent disease, while others have evaluated intraoperative intravascular ultrasound evaluation of luminal diameters to be predictors of disease recurrence with no difference in the outcome with stent type (Wallstent Boston Scientific or Wallstent-Z stent Cook Medical), but rather significant recurrence of symptoms with more severe degree (>90\%) of stenosis. ${ }^{14,15}$ Authors strongly feel that health care practitioners (and in particular primary care and emergency physicians) should be aware of this vascular compression syndrome and its association with increased risk of recurrent or persistent DVT particularly in young women, and the role of prompt and successful endovascular intervention in relieving root cause problem in such patients.

Study limitations. The retrospective nature of the study, single-center, and small sample size. We did not look at the right sided MTS in bilateral or right sided DVTs. Also, asymptomatic MTS (better called as MTS phenomenon) cases were not considered or followed.

Large scale studies are needed to address long-term benefits of endovascular interventions in sub-groups of MTS (those having hypercoagulable states, unrecognized or delayed MTS, and MTS in asymptomatic patients) to recognize both clinical and imaging predictors of failed interventions or complications (in-stent restenosis).

In conclusion, patients with MTS as cause of left sided DVTs may benefit from early endovascular interventions.
Acknowledgment. The authors gratefully acknowledge Dr. Muhammad Z. Ul Hassan for providing his assistance in reviewing the submitted figures for clarity and correctness. We also would like to thank American Manuscript Editors (www.americanmanuscripteditors.com) for English language editing.

Received 12th June 2021. Accepted 5th October 2021.

From the Department of Radiology (Waheed, Salem, Alshehri, Said, Almubarak); from the Department of Vascular Surgery (Mohammed, Shaltout), King Fahad Military Medical Complex, and from the Vice Deanship of Post-graduate and Research (Arulanantham), Prince Sultan Military College of Health Science, Dhahran, Kingdom of Saudi Arabia.

Address correspondence and reprints request to: Dr. Khawaja B. Waheed, Consultant Radiologist, Radiology Department, King Fahad Military Medical Complex, Dhahran, Kingdom of Saudi Arabia.E-mail: khawaja@kfmmc.med.sa ORCID ID: https://orcid.org/0000-0001-6340-3139

\section{References}

1. Monreal M, Agnelli G, Chuang LH, Cohen AT, Gumbs PD, Bauersachs R, et al. Deep vein thrombosis in Europe-healthrelated quality of life and mortality. Clin Appl Thromb Hemost 2019; 25: 1076029619883946.

2. Hansrani V, Moughal S, Elmetwally A, Al-Khaffaf H. A review into the management of May-Thurner syndrome in adolescents. J Vasc Surg Venous Lymphat Disord 2020; 8: 1104-1110.

3. Virchow R. [Ueber die Erweiterung kleinerer Gefäfse]. Archiv f pathol Anat 1851; 3: 427-462. [in Germen]

4. Liddell RP, Evans NS. May-Thurner syndrome. Vasc Med 2018; 23: 493-496.

5. Mako K, Puskas A. May-Thurner syndrome - Are we aware enough? Vasa 2019; 48: 381-388.

6. Knuttinen MG, Naidu S, Oklu R, Kriegshauser S, Eversman W, Rotellini L, et al. May-Thurner: diagnosis and endovascular management. Cardiovasc Diagn Ther 2017; 7: S159-S164.

7. Jin S, Sun Z, Li X, Jian T, Jin X, Li S, et al. May-Thurner syndrome and the risk of pulmonary embolism in patients with acute deep venous thrombosis. J Vasc Surg Venous Lymphat Disord 2018; 6: 433-440.

8. Jeanneret C. May-Thurner syndrome. Vasa 2019; 48: 377-378.

9. Kaltenmeier CT, Erben Y, Indes J, Lee A, Dardik A, Sarac T, et al. Systematic review of May-Thurner syndrome with emphasis on gender differences. J Vasc Surg Venous Lymphat Disord 2018; 6: 399-407.

10. Sedhai YR, Golamari R, Salei A, Alukal J, Basnyat S, Pathak S, et al. May-Thurner syndrome. Am J Med Sci 2018; 355: 510-514.

11. Yamamoto K, Gondo G, Ogino H, Watanabe T, Tanaka M, Tanaka S, et al. Sciatic neuralgia caused by May-Thurner syndrome. World Neurosurg 2018; 116: 40-43.

12. Chang SC, Tsai MJ, Hsu CF. May-Thurner syndrome caused by a huge uterine myoma. Ci Ji Yi Xue Za Zhi 2017; 29: 235-236.

13. Mei JY, Deshmukh U, Negi M, Campbell K, Paidas MJ, Platt LD, et al. May-Thurner syndrome in pregnancy: a multiinstitutional case series and review of the literature. Am J Obstet Gynecol MFM 2020; 2: 100240. 
14. J Padrnos L, Garcia D. May-Thurner syndrome and thrombosis: a systematic review of antithrombotic use after endovascular stent placement. Res Pract Thromb Haemost 2018; 3: 70-78.
15. Bondarev S, Keller EJ, Han T, Young VA, Gupta S, Vogelzang $\mathrm{RL}$, et al. Predictors of disease recurrence after venoplasty and stent placement for May-Thurner syndrome. J Vasc Interv Radiol 2019; 30: 1549-1554. 